\title{
Myeloid Neoplasms with Germline Predisposition and Preexisting Platelet Disorders
}

National Cancer Institute

\section{Source}

National Cancer Institute. Myeloid Neoplasms with Germline Predisposition and

Preexisting Platelet Disorders. NCI Thesaurus. Code C151902.

Myeloid neoplasms associated with germline mutations and familial platelet disorders. 